$\mathrm{J}$ o u r n a l of

Mathematics

and Applications

JMA No 42, pp 5-19 (2019)

\title{
Fixed Point Theorems for Monotone Mappings in Ordered Banach Spaces Under Weak Topology Features
}

\author{
Abdullah Alahmari, Mohamed Mabrouk and \\ Mohamed-Aziz Taoudi
}

\begin{abstract}
We present several fixed point theorems for monotone nonlinear operators in ordered Banach spaces. The main assumptions of our results are formulated in terms of the weak topology. As an application, we study the existence of solutions to a class of first-order vectorvalued ordinary differential equations. Our conclusions generalize many well-known results.
\end{abstract}

AMS Subject Classification: 45N05, 47H10.

Keywords and Phrases: Fixed point theorem; Order cone; Increasing operator; Decreasing operator; Weakly condensing; Measure of weak noncompactness.

\section{Introduction}

Fixed point theory furnishes an effective and important tool for proving theoretical as well as constructive existence for a variety of nonlinear problems arising from the mathematical modelling of real world phenomena. The usual topological fixed point methods (Schauder, Darbo, Sadovskii,...) are generally only suited to nonlinear problems with continuity and compactness. However, many problems in theory and applications have no compactness. Some attempts have been made to overcome this difficulty by using the weak topology, see $[2,3,6,7,8,9,10,11,14,34]$. The interest of the weak topology is mainly due to the vital role played by weak compactness in the theory of infinite dimensional linear spaces. In particular, a Banach space $\mathrm{X}$ is reflexive if and only if the closed unit ball is weakly compact. Equally, fixed point theorems using the weak topology (Schauder-Tychonov, Arino-Gautier-Penot,...) are

COPYRIGHT (c) by Publishing House of Rzeszów University of Technology P.O. Box 85, 35-959 Rzeszów, Poland 
generally only suited to nonlinear problems with weak (sequential) continuity and weak compactness. In several situations, the weak (sequential) continuity could rise several difficulties. For example, in $L^{1}$-spaces, which are the most natural functional settings of many real world problems in physics and population dynamics (notably when the unknown is a density), only linear superposition (Nemytskii) operators are weakly (sequentially) continuous [4]. To our knowledge, the first paper where the weak topology was successfully applied to fixed point theorems without requiring the weak continuity of the involved operators, was [29]. In the quoted paper, the authors used the concepts of ws-compactness and ww-compactness instead of the (sequential) weak continuity. Such concepts proved to be more effective in many practical situations especially when we work in nonreflexive Banach spaces. This fact was illustrated by proving the existence of an integrable solution for a stationary nonlinear problem arising in transport theory and kinetic of gas and in many other situations $[12,13,16,20,21,22,29,30]$.

In the present paper, we provide a new general treatment of fixed point theory of monotone mappings in ordered vector spaces. Specifically, we will show how weak topology is successfully used in conjunction with the order in fixed point problems. As the functional setting of many nonlinear problems arising from the mathematical modeling of real world phenomena is usually an ordered vector space, our approach gives an extremely powerful and direct tool to investigate the solvability of a large class of evolution equations with lack of compactness. To illustrate our results, we investigate the solvability of a class of first-order vector-valued ordinary differential equations. Before proceeding to the detailed discussion, we recall some related definitions and auxiliary results. Let $X$ be a Banach space and let $P$ be a subset of $X$. The set $P$ is called an order cone if and only if:

(i) $P$ is closed, nonempty and $P \neq\{0\}$,

(ii) $a, b \in \mathbb{R}, a, b \geq 0, x, y \in P \Rightarrow a x+b y \in P$,

(iii) $x \in P$ and $-x \in P \Rightarrow x=0$.

An order cone permits to define a partial order in $X$ by

$$
x \leq y \text { iff } y-x \in P .
$$

Conversely, let $X$ be a real Banach space with a partial order compatible with the algebraic operations in $X$, that is,

$$
\begin{gathered}
x \geq 0 \text { and } \lambda \geq 0 \text { implies } \lambda x \geq 0 \\
x_{1} \leq y_{1} \text { and } x_{2} \leq y_{2} \text { implies } x_{1}+x_{2} \leq y_{1}+y_{2} .
\end{gathered}
$$

The positive cone of $X$ is defined by

$$
X^{+}=\{x \in X: 0 \leq x\} .
$$




\section{Definition 1.1.}

(i) A subset $M \subset X$ is said order bounded if there exist $u, v \in X$ such that $u \leq x \leq v$, for all $x \in M$.

(ii) The order cone $P$ is called normal if and only if there is a number $c>0$ such that for all $x, y \in X$ we have

$$
0 \leq x \leq y \Rightarrow\|x\| \leq c\|y\|
$$

The least positive number $c$ (if it exists) satisfying (1.1) is called a normal constant.

Remark 1.2. If the cone $P$ is normal, then every order interval is norm bounded (see e.g. [23, Theorem 2.1.1]).

Remark 1.3. Let $K$ be a compact Hausdorff space and $E$ be an ordered Banach space with normal positive cone. We denote by $C(K, E)$ the Banach space of all continuous $E$-valued functions on $K$ endowed with the usual maximum norm. Plainly $C(K, E)$ is an ordered Banach space with the natural ordering whose positive cone is given by

$$
C^{+}(K, E)=\left\{f \in C(K, E): f(x) \in E^{+}, \forall x \in K\right\} .
$$

Since $E^{+}$is normal so is $C^{+}(K, E)$.

The following definitions are frequently used in the sequel.

Definition 1.4. Let $M \subset X$. The operator $T: M \rightarrow X$ is said to be an increasing operator if $x, y \in M, x \leq y$ implies $T x \leq T y$. The operator $T: M \rightarrow X$ is said to be a decreasing operator if $x, y \in M, x \leq y$ implies $T y \leq T x$.

Definition 1.5. Let $M$ be a nonempty closed subset of $X$. The operator $T: M \rightarrow X$ is said to be monotone-subcontinuous if for any monotone sequence (increasing or decreasing) $\left(x_{n}\right)$ in $M$ that converges strongly to $x$ the sequence $\left(T x_{n}\right)$ converges weakly to $T x$.

The following elementary result serves as the key tool in the proof of more sophisticated results.

Lemma 1.6. [26] Let $X$ be an ordered real Banach space with a normal order cone. Suppose that $\left\{x_{n}\right\}$ is a monotone sequence which has a subsequence $\left\{x_{n_{k}}\right\}$ converging weakly to $x_{\infty}$. Then $\left\{x_{n}\right\}$ converges strongly to $x_{\infty}$. Moreover, if $\left\{x_{n}\right\}$ is an increasing sequence, then $x_{n} \leq x_{\infty}(n=1,2,3, \ldots)$; if $\left\{x_{n}\right\}$ is a decreasing sequence, then $x_{\infty} \leq x_{n}(n=1,2,3, \ldots)$.

By a poset $F=(F, \leq)$ we mean a nonempty set $F$ equipped with a partial ordering relation $\leq$

Lemma 1.7. [25, Lemma 1.1.5] Let $\left\{x_{n}\right\}$ be a sequence in a poset F. 
(a) If $\left\{x_{n}\right\}$ is totally ordered, then it has a monotone subsequence.

(b) If $\left\{x_{n}\right\}$ is nondecreasing (resp. nonincreasing), then it has the supremum (resp. the infimum) $x$ if and only if $x$ is the supremum (resp. the infimum) of some of its subsequences.

Combining Lemma 1.6 and Lemma 1.7 we obtain the following interesting result.

Lemma 1.8. Let $X$ be an ordered real Banach space with a normal order cone. Suppose that $\left\{x_{n}\right\}$ is a totally ordered sequence which is contained in a relatively weakly compact set. Then $\left\{x_{n}\right\}$ converges strongly in $X$.

In what follows, $\psi$ will always denote a measure of weak noncompactness (MWNC) on the Banach space $X$. We refer the reader to [5] for the axiomatic definition of a measure of weak noncompactness. One of the most frequently exploited measure of weak noncompactness was defined by De Blasi [15] as follows:

$$
w(M)=\inf \left\{r>0: \text { there exists } W \text { weakly compact such that } M \subseteq W+B_{r}\right\},
$$

for each bounded subset $M$ of $X$; Here, $B_{r}$ stands for the closed ball of $X$ centered at origin with radius $r$.

The following results are crucial for our purposes. We first state a theorem of Ambrosetti type (see [31] for a proof).

Theorem 1.9. Let $E$ be a Banach space and let $H \subseteq C([0, T], E)$ be bounded and equicontinuous. Then the map $t \rightarrow w(H(t))$ is continuous on $[0, T]$ and

$$
w(H)=\sup _{t \in[0, T]} w(H(t))=w(H[0, T])
$$

where $H(t)=\{h(t): h \in H\}$ and $H[0, T]=\bigcup_{t \in[0, T]}\{h(t): h \in H\}$.

The following Lemma is well-known (see for example [32]).

Lemma 1.10. If $H \subseteq C([0, T], E)$ is equicontinuous and $x_{0} \in C([0, T], E)$, then $\overline{c o}\left(H \cup\left\{x_{0}\right\}\right)$ is also equicontinuous in $C([0, T], E)$.

\section{Fixed point results}

In this section, we prove some fixed point theorems for monotone mappings in ordered Banach spaces. Our results combine the advantages of the strong topology (i.e. the involved mappings will be continuous (or subcontinuous) with respect to the strong topology) with the advantages of the weak topology (i.e. the maps will satisfy some compactness conditions relative to the weak topology) to draw new conclusions about fixed points for a given monotone map.

Theorem 2.1. Let $X$ be an ordered Banach space with a normal cone P. Let $u_{0}, v_{0} \in$ $X$ with $u_{0}<v_{0}$ and $A:\left[u_{0}, v_{0}\right] \rightarrow X$ be a monotone-subcontinuous increasing operator satisfying the following: 


$$
u_{0} \leq A u_{0}, A v_{0} \leq v_{0}
$$

If, in addition, $A$ verifies

$\left(\mathcal{P}\left(n_{0}\right)\right):$ There exists an integer $n_{0} \geq 1$ such that: for any monotone sequence $V=\left\{x_{n}\right\}$ of $\left[u_{0}, v_{0}\right]$ and any finite subset $F$ of $\left[u_{0}, v_{0}\right]$ of cardinal $n_{0}$, we have:

$$
V=F \cup A^{n_{0}}(V) \text { implies } V \text { is relatively weakly compact. }
$$

Then, $A$ has a minimal fixed point $u_{*}$ and a maximal fixed point $u^{*}$ in $\left[u_{0}, v_{0}\right]$ and

$$
u_{*}=\lim _{n \rightarrow \infty} u_{n} \text { and } u^{*}=\lim _{n \rightarrow \infty} v_{n}
$$

where $u_{n}=A u_{n-1}$ and $v_{n}=A v_{n-1}, n=1,2, \ldots$

$$
u_{0} \leq u_{1} \leq \cdots u_{*} \leq u^{*} \leq \cdots \leq v_{n} \leq \cdots \leq v_{1} \leq v_{0} .
$$

Proof. Let $u_{n}=A u_{n-1}$ and $v_{n}=A v_{n-1}$ for $n \geq 1$. Since $A$ is increasing, then

$$
u_{0} \leq u_{1} \leq \cdots \leq u_{n} \leq \cdots v_{n} \leq \cdots v_{1} \leq v_{0}
$$

Let $S=\left\{u_{0}, u_{1}, \ldots, u_{n}, \ldots\right\}$. Clearly, for any integer $k \geq 1$ we have

$$
A^{k}(S) \cup\left\{u_{0}, u_{1}, \ldots, u_{k-1}\right\}=S .
$$

From our hypotheses we know that $S$ is relatively weakly compact. Referring to Lemma 1.8 , we see that $\left\{u_{n}\right\}$ is convergent. Let $u_{*}$ be its limit. The monotonesubcontinuity of $A$ yields $A u_{*}=u_{*}$. Similarly, we can prove that $\left\{v_{n}\right\}$ converges to some $u^{*}$ and $A u^{*}=u^{*}$. Finally, we prove that $u^{*}$ and $u_{*}$ are the maximal and minimal fixed points of $A$ in $\left[u_{0}, v_{0}\right]$. Let $x \in\left[u_{0}, v_{0}\right]$ and $A x=x$. Since $A$ is increasing, it follows from $u_{0} \leq x \leq v_{0}$ that $A u_{0} \leq A x \leq A v_{0}$, i.e. $u_{1} \leq x \leq v_{1}$. Using the same argument, we get $u_{2} \leq x \leq v_{2}$ and, in general, $u_{n} \leq x \leq v_{n}(n=1,2,3, \ldots)$. Now, letting $n$ go to infinity we get $u_{*} \leq x \leq u^{*}$.

As a convenient specialization of Theorem 2.1, we state the following.

Corollary 2.2. Let $X$ be an ordered Banach space with a normal cone $P$. Let $u_{0}, v_{0} \in X$ with $u_{0}<v_{0}$ and $A:\left[u_{0}, v_{0}\right] \rightarrow X$ be a monotone-subcontinuous increasing operator satisfying the following:

$$
u_{0} \leq A u_{0}, A v_{0} \leq v_{0} .
$$

If, in addition, $A$ verifies

$(\mathcal{P}(1)):$ if $V=\left\{x_{n}\right\}$ is a monotone sequence of $\left[u_{0}, v_{0}\right]$ and $a \in\left[u_{0}, v_{0}\right]$, then $V=\{a\} \cup A(V)$ implies $V$ is relatively weakly compact.

Then $A$ has a minimal fixed point $u_{*}$ and a maximal fixed point $u^{*}$ in $\left[u_{0}, v_{0}\right]$ satisfying (2.2) and (2.3). 
Proof. Apply Theorem 2.1 with $n_{0}=1$.

Another consequence of Theorem 2.1 is the following. Recall that a measure of weak noncompactness $\psi$ on a Banach space $X$ is said to be nonsingular if $\psi(M \cup\{a\})=\psi(M)$ for every $a \in X$ and every nonempty bounded subset $M$ of $X$.

Corollary 2.3. Let $X$ be an ordered Banach space with a normal cone $P$ and $\psi$ be a nonsingular measure of weak noncompactness on $X$. Let $u_{0}, v_{0} \in X$ with $u_{0}<v_{0}$ and $A:\left[u_{0}, v_{0}\right] \rightarrow X$ be a monotone-subcontinuous increasing operator satisfying the following:

$$
u_{0} \leq A u_{0}, A v_{0} \leq v_{0} .
$$

In addition, if for any $\Omega=\left\{u_{n}\right\} \subset\left[u_{0}, v_{0}\right]$ countable and monotone with $\psi(\Omega) \neq 0$ we have

$$
\psi\left(A^{n_{0}}(\Omega)\right)<\psi(\Omega),
$$

for some integer $n_{0} \geq 1$. Then, $A$ has a minimal fixed point $u_{*}$ and a maximal fixed point $u^{*}$ in $\left[u_{0}, v_{0}\right]$ satisfying (2.2) and (2.3).

Proof. By virtue of Theorem 2.1, it suffices to show that $\left(\mathcal{P}\left(n_{0}\right)\right)$ holds true. To do this, let $V=\left\{x_{n}\right\}$ be a monotone sequence of $\left[u_{0}, v_{0}\right]$ and $F$ be a finite subset of $\left[u_{0}, v_{0}\right]$ of cardinal $n_{0}$ such that $V=F \cup A^{n_{0}}(V)$. Since $P$ is normal then, according to Remark 1.2, the order interval $\left[u_{0}, v_{0}\right]$ is bounded. This implies that $V$ and $A^{n_{0}}(V)$ are bounded and we have $\psi(V)=\psi\left(F \cup A^{n_{0}}(V)\right)=\psi\left(A^{n_{0}}(V)\right)$. Consequently, it follows from our hypotheses that $\psi(V)=0$, which means that $V$ is relatively weakly compact. This achieves the proof.

Remark 2.4. Corollary 2.3 extends [23, Theorem 3.1.1].

Corollary 2.5. Let $u_{0}, v_{0} \in X$ with $u_{0}<v_{0}$ and $A:\left[u_{0}, v_{0}\right] \rightarrow X$ be a monotonesubcontinuous increasing operator satisfying (2.6). If $P$ is normal and $A^{n_{0}}\left(\left[u_{0}, v_{0}\right]\right)$ is relatively weakly compact for some integer $n_{0} \geq 1$, then $A$ has a minimal fixed point $u_{*}$ and a maximal fixed point $u^{*}$ in $\left[u_{0}, v_{0}\right]$ satisfying (2.2) and (2.3).

For later use, we consider the following condition.

$(\mathcal{C})\left\{\begin{array}{l}A: P \rightarrow P \text { satisfies } A^{2} \theta \geq \epsilon A \theta \text { where } 0<\epsilon<1 \text {, and for any } \\ \epsilon A \theta \leq x \leq A \theta \text { and } \epsilon \leq t<1, \text { there exists } \eta=\eta(x, t)>0 \text {, such that } \\ A(t x) \leq(t(1+\eta))^{-1} A x .\end{array}\right.$

We will need the following lemmas from [23].

Lemma 2.6. [23, Lemma 3.2.1] Let $A: P \rightarrow P$ be a decreasing operator satisfying the condition $(\mathcal{C})$. If $u, v \in P$ with $A u=v$ and $A v=u$, then $u=v$.

Lemma 2.7. [23, Lemma 3.2.2] Let $A: P \rightarrow P$ be a decreasing operator satisfying the condition $(\mathcal{C})$. If $u, v \in P$ with $A u=u$ and $A v=v$, then $u=v$. 
Theorem 2.8. Let $X$ be an ordered Banach space with a normal cone $P$. Let $A: P \rightarrow P$ be a monotone-subcontinuous decreasing operator satisfying the conditions $(\mathcal{C})$ and $\left(\mathcal{P}\left(n_{0}\right)\right)$ for some integer $n_{0} \geq 1$. Then $A$ has a unique fixed point $u^{*}$ in $P$ and

$$
u^{*}=\lim _{n \rightarrow \infty} u_{n}
$$

where $u_{n}=A u_{n-1}, n=1,2, \ldots$

Proof. Keeping in mind that $A: P \rightarrow P$ is decreasing we easily deduce that

$$
\theta=u_{0} \leq u_{2} \leq \cdots \leq u_{2 n} \leq \cdots \leq u_{2 n+1} \leq \cdots \leq u_{1}=A \theta
$$

Let $S=\left\{u_{0}, u_{1}, \ldots, u_{n}, \ldots\right\}$. From (2.8) and the normality of $P$ we infer that $S$ is bounded. Clearly, for any integer $k \geq 1$ we have

$$
A^{k}(S) \cup\left\{u_{0}, u_{1}, \ldots, u_{k-1}\right\}=S .
$$

From our hypotheses we know that $S$ is relatively weakly compact. This implies that the increasing sequence $\left\{u_{2 n}\right\}$ has a weakly convergent subsequence. Referring to Lemma 1.6, we see that $\left\{u_{2 n}\right\}$ is convergent. Let $u_{*}$ be its limit. Similarly we can prove that the sequence $\left\{u_{2 n+1}\right\}$ converges to some $u^{*}$. Taking the limit at the both sides of $u_{2 n+1}=A u_{2 n}$ and $u_{2 n+2}=A u_{2 n+1}$ and using the monotone-subcontinuity of $A$ we get $u_{*} \leq u^{*}, u^{*}=A u_{*}$ and $u^{*}=A u_{*}$. Invoking Lemma 2.6 we infer that $u^{*}=u_{*}$ is a fixed point of $A$. The uniqueness follows from Lemma 2.7.

As a convenient specialization of Theorem 2.8 we obtain the following result.

Corollary 2.9. Let $X$ be an ordered Banach space with a normal cone $P$ and $\psi$ be a nonsingular measure of weak noncompactness on $X$. Let $A: P \rightarrow P$ be a monotonesubcontinuous decreasing operator satisfying the condition $(\mathcal{C})$. In addition, if for any $\Omega=\left\{u_{n}\right\} \subset P$ countable and monotone with $\psi(\Omega) \neq 0$ we have

$$
\psi\left(A^{n_{0}}(\Omega)\right)<\psi(\Omega)
$$

for some integer $n_{0} \geq 1$, then $A$ has a unique fixed point $u^{*}$ in $P$ and

$$
u^{*}=\lim _{n \rightarrow \infty} u_{n}
$$

where $u_{n}=A u_{n-1}, n=1,2, \ldots$

Proof. In view of Theorem 2.8, it suffices to show that $A$ verifies $\left(\mathcal{P}\left(n_{0}\right)\right)$. The reasoning in Corollary 2.3 yields the result.

Remark 2.10. Theorem 2.8 and Corollary 2.9 extend [23, Theorem 3.2.1]. 


\section{Application to differential equations}

We shall use the results in previous sections to get an existence theorem for a nonlinear ODE in a Banach space. The nonlinear term satisfies an appropriate condition expressed in terms of the De Blasi measure of weak noncompactness. Let $E$ be an ordered Banach space with a normal cone $P$. We consider the following initial value problem

$$
u^{\prime}=f(t, u) \text { on } I, u(0)=u_{0},
$$

where $I=[0,1], u \in C^{1}(I, E), f \in C(I \times E, E)$. A vector-valued function $u: I \rightarrow E$ is said to be a solution of (3.1) on $I$ if $u(t)$ is continuously differentiable and satisfies (3.1) on $I$.

In [18], Du and Lakshmikantham proved that if the problem (3.1) has a lower solution $v_{0}$ and an upper solution $w_{0}$ with $v_{0} \leq w_{0}$, and the nonlinear term satisfies the monotonicity condition

$$
f(t, y)-f(t, x) \geq-M(y-x) \text { whenever } v_{0}(t) \leq x \leq y \leq w_{0}(t)
$$

for some $M>0$, and the compactness measure condition

$$
\alpha(f(t, V)) \leq \tau \alpha(V)
$$

for any $t \in I$ and any bounded subset $V$ of $E$, where $\tau$ is a positive constant and $\alpha($.$) denotes the Kuratowski measure of noncompactness in E$, then the problem (3.1) has a minimal and a maximal solution between $v_{0}$ and $w_{0}$, which can be obtained by a monotone iterative procedure starting from $v_{0}$ and $w_{0}$ respectively. When $E$ is weakly sequentially complete, Y. Du [17] improved the result of [18] and removed the condition (3.3).

Our aim in this section is to improve and extend the aforementioned results. We will replace the noncompactness measure condition (3.3) by a weaker condition expressed in terms of the De Blasi measure of weak noncompactness. From now on, we assume the following:

(i) There exist $v_{0}, w_{0} \in C^{1}(I, E)$ with $v_{0}(t) \leq w_{0}(t)$ on $I$ such that:

$$
\begin{gathered}
v_{0}^{\prime}(t) \leq f\left(t, v_{0}(t)\right), v_{0}(0) \leq u_{0} \\
w_{0}^{\prime}(t) \geq f\left(t, w_{0}(t)\right), w_{0}(0) \geq u_{0} .
\end{gathered}
$$

(ii) For some $M>0$,

$$
f(t, y)-f(t, x) \geq-M(y-x) \text { whenever } v_{0}(t) \leq x \leq y \leq w_{0}(t) .
$$


(iii) There is a constant $\tau \geq 0$ such that for any equicontinuous monotone sequence $V=\left\{u_{n}\right\}$ of $\left[v_{0}, w_{0}\right]$ and for any $a, b \in[0,1]$ with $a<b$ we have

$$
w(f([a, b] \times V)) \leq \tau w(V[a, b]),
$$

where $f([a, b] \times V):=\{f(s, x(s)), a \leq s \leq b, x \in V\}$.

Remark 3.1. Let $g(s, x)=f(s, x)+M x$. Then, for any monotone sequence $V=\left\{u_{n}\right\}$ of $\left[v_{0}, w_{0}\right]$ and for any $a, b \in[0,1]$ with $a<b$ we have

$$
w(g([a, b] \times V)) \leq \mu w(V[a, b]),
$$

where $\mu=\tau+M$.

Now, let $t \in[0,1]$ be fixed and let $h(s, x)=e^{-M(t-s)} g(s, x)$, for $s \in[0, t]$ and $x \in E$. It is readily verified that

$$
h([0, t] \times V) \subset \operatorname{co}(g([0, t] \times V) \cup\{0\}) .
$$

Combining (3.4) and (3.5) we arrive at

$$
w(h([0, t] \times V)) \leq \mu w(V[0, t]),
$$

where $h([0, t] \times V):=\{h(s, x(s)), 0 \leq s \leq t, x \in V\}$.

Now, we are in a position to state our main result.

Theorem 3.2. Let assumptions (i)-(iii) be satisfied. Then the problem (3.1) has a maximal and a minimal solution between $v_{0}$ and $w_{0}$, which can be obtained by a monotone iterative procedure starting from $v_{0}$ and $w_{0}$ respectively.

Proof. We consider the equivalent modified problem

$$
u^{\prime}+M u=f(t, u)+M u \text { on } I, u(0)=u_{0},
$$

which is equivalent to the problem

$$
\left(e^{M t} u\right)^{\prime}=e^{M t}(f(t, u)+M u) \text { on } I, u(0)=u_{0} .
$$

Let us write (3.8) as an integral equation

$$
u(t)=e^{-M t} u_{0}+\int_{0}^{t} e^{-M(t-s)}(f(s, u(s))+M u(s)) d s .
$$

Define the operator $A$ on $C(I, E)$ by

$$
(A u)(t)=e^{-M t} u_{0}+\int_{0}^{t} e^{-M(t-s)}(f(s, u(s))+M u(s)) d s, t \in I .
$$


It is easy to check that a fixed point of $A$ is a solution of (3.1). We will demonstrate that $A$ satisfies all the hypotheses of Theorem 2.1. It is apparent that $A$ is continuous. From Hypothesis (ii) we know that $A$ is increasing on $\left[v_{0}, w_{0}\right]$. To illustrate that $v_{0} \leq A v_{0}$, let $k(t)=v_{0}^{\prime}(t)+M v_{0}(t)$. Clearly, $k \in C(I, E)$ and $k(t) \leq f\left(t, v_{0}(t)\right)+$ $M v_{0}(t), t \in I$. Keeping in mind the fact that $\left(e^{M t} v_{0}(t)\right)^{\prime}=e^{M t} k(t)$, we deduce that for all $t \in I$ we have:

$$
\begin{aligned}
e^{M t} v_{0}(t) & =v_{0}(0)+\int_{0}^{t} e^{M s} k(s) d s \\
& \leq u_{0}+\int_{0}^{t} e^{M s}\left(f\left(s, v_{0}(s)\right)+M v_{0}(s)\right) d s
\end{aligned}
$$

Accordingly, $v_{0} \leq A v_{0}$. Similarly, we can prove that $A w_{0} \leq w_{0}$. We claim that for any integer $k \geq 1$ and any $V \subset\left[u_{0}, v_{0}\right]$ the set $A^{k}(V)$ is equicontinuous. Indeed, let $t, t_{0} \in I$ with $t<t_{0}$ and $u \in\left[v_{0}, w_{0}\right]$. Then,

$$
\begin{aligned}
\left\|A u(t)-A u\left(t_{0}\right)\right\| \leq & \left(e^{-M t}-e^{-M t_{0}}\right)\left\|u_{0}\right\|+\int_{0}^{t}\left(e^{-M(t-s)}-e^{-M\left(t_{0}-s\right)}\right)\|g(s, u(s))\| d s \\
& +\int_{t}^{t_{0}}\|g(s, u(s))\| d s .
\end{aligned}
$$

For any $u \in\left[v_{0}, w_{0}\right]$, by Assumption (ii), we have

$$
g\left(s, v_{0}(s)\right) \leq g(s, u(s)) \leq g\left(s, w_{0}(s)\right) .
$$

By the normality of the cone $P$, there exists $C_{g}>0$ such that

$$
\|g(t, u(t))\| \leq C_{g}, u \in\left[v_{0}, w_{0}\right] .
$$

Accordingly,

$$
\begin{aligned}
\left\|A u(t)-A u\left(t_{0}\right)\right\| \leq & \left(e^{-M t}-e^{-M t_{0}}\right)\left\|u_{0}\right\|+C_{g} \int_{0}^{t}\left(e^{-M(t-s)}-e^{-M\left(t_{0}-s\right)}\right) d s \\
& +C_{g}\left(t_{0}-t\right) .
\end{aligned}
$$

Consequently,

$$
\left\|A u(t)-A u\left(t_{0}\right)\right\| \rightarrow 0 \text { as } t \rightarrow t_{0}^{-},
$$

uniformly with respect to $u$. Similarly, we get

$$
\left\|A u(t)-A u\left(t_{0}\right)\right\| \rightarrow 0 \text { as } t \rightarrow t_{0}^{+},
$$

uniformly with respect to $u$. This proves that $A(V)$ is equicontinuous. Therefore, for any integer $k \geq 1$ the set $A^{k}(V)$ is equicontinuous.

Now, let $V \subset\left[v_{0}, w_{0}\right]$ and $F$ be a finite subset of $\left[v_{0}, w_{0}\right]$ such that $V=A^{k}(V) \cup F$, for some integer $k \geq 1$. Since $A^{k}(V)$ is equicontinuous, then by invoking Lemma 1.10 
we conclude that $V$ is equicontinuous. Let $h$ be as described in Remark 3.1, then for each $t \in I$, we have

$$
\begin{aligned}
w(A(V)(t)) & =w\left(\left\{e^{-M t} u_{0}+\int_{0}^{t} h(s, u(s)) d s: u \in V\right\}\right) \\
& \leq w(t \overline{c o}\{h(s, u(s)): u \in V, s \in[0, t]\}) \\
& =t w(\overline{c o}\{h(s, u(s)): u \in V, s \in[0, t]\}) \\
& \leq t w(h([0, t] \times V) \\
& \leq t \mu w(V[0, t]) .
\end{aligned}
$$

Theorem 1.9 implies (since $V$ is equicontinuous) that

$$
w(A(V)(t)) \leq t \mu w(V) .
$$

Using (3.11) we get

$$
\begin{aligned}
w\left(A^{2}(V)(t)\right) & =w\left(\left\{e^{-M t} u_{0}+\int_{0}^{t} h(s, u(s)) d s: u \in A(V)\right\}\right) \\
& =w\left(\left\{\int_{0}^{t} h(s, u(s)) d s: u \in A(V)\right\}\right) .
\end{aligned}
$$

Fix $t \in[0,1]$. We divide the interval $[0, t]$ into $m$ parts $0=t_{0}<t_{1}<\cdots<t_{m}=t$ in such a way that $\Delta t_{i}=t_{i}-t_{i-1}=\frac{t}{m}, i=1, \ldots, m$. For each $u \in A(V)$ we have

$$
\begin{aligned}
\int_{0}^{t} h(s, u(s)) d s & =\sum_{i=1}^{m} \int_{t_{i-1}}^{t_{i}} h(s, u(s)) d s \\
& \in \sum_{i=1}^{m} \Delta t_{i} \overline{c o}\left\{h(s, u(s)): u \in A(V), s \in\left[t_{i-1}, t_{i}\right]\right\} \\
& \subseteq \sum_{i=1}^{m} \Delta t_{i} \overline{c o}\left(h\left(\left[t_{i-1}, t_{i}\right] \times A(V)\right) .\right.
\end{aligned}
$$

Using again Theorem 1.9 we infer that for each $i=2, \ldots, m$ there is a $s_{i} \in\left[t_{i-1}, t_{i}\right]$ such that

$$
\sup _{s \in\left[t_{i-1}, t_{i}\right]} w(A(V)(s))=w\left(A(V)\left[t_{i-1}, t_{i}\right]\right)=w\left(A(V)\left(s_{i}\right)\right) .
$$

Consequently

$$
\begin{aligned}
w\left(\left\{\int_{0}^{t} h(s, x(s)) d s: u \in A(V)\right\}\right. & \leq \sum_{i=1}^{m} \Delta t_{i} w\left(\overline{c o}\left(h\left(\left[t_{i-1}, t_{i}\right] \times A(V)\right)\right)\right. \\
& \leq \mu \sum_{i=1}^{m} \Delta t_{i} w\left(\left(A(V)\left(\left[t_{i-1}, t_{i}\right]\right)\right)\right. \\
& \leq \mu \sum_{i=1}^{m} \Delta t_{i} w\left(A(V)\left(\left(s_{i}\right)\right) .\right.
\end{aligned}
$$


On the other hand, if $m \rightarrow \infty$ then

$$
\sum_{i=1}^{m} \Delta t_{i} w\left(A(V)\left(\left(s_{i}\right)\right) \rightarrow \int_{0}^{t} w(A(V)(s)) d s\right.
$$

As a result,

$$
w\left(A^{2}(V)(t)\right) \leq \frac{(\mu t)^{2}}{2} w(V) .
$$

By induction we get

$$
w\left(A^{n}(V)(t)\right) \leq \frac{(\mu t)^{n}}{n !} w(V) .
$$

Invoking Theorem 1.9 we obtain

$$
w\left(A^{n}(V)\right) \leq \frac{\mu^{n}}{n !} w(V) .
$$

Since $\lim _{n \rightarrow \infty} \frac{\mu^{n}}{n !}=0$, we may choose $n_{0}$ as large as we please such that $\frac{\mu^{n_{0}}}{n_{0} !}<1$.

Now, let $V \subset\left[v_{0}, w_{0}\right]$ and $F$ be a finite subset of $\left[v_{0}, w_{0}\right]$ such that $V=A^{n_{0}}(V) \cup F$. Then, $w(V)=w\left(A^{n_{0}}(V) \cup F\right)=w\left(A^{n_{0}}(V)\right) \leq \frac{\mu^{n_{0}}}{n_{0} !} w(V)$. Thus, $w(V)=0$ and therefore $V$ is relatively weakly compact. By applying Theorem 2.1 we infer that $A$ has a maximal and a minimal fixed points between $v_{0}$ and $w_{0}$, which can be obtained by a monotone iterative procedure starting from $v_{0}$ and $w_{0}$ respectively. This completes the proof.

Remark 3.3. If $E$ is weakly sequentially complete (reflexive, in particular), then the condition (iii) in Theorem 3.2 holds automatically. In fact, according to [17, Theorem 2.2 ] any monotone order-bounded sequence is relatively compact. Thus, Theorem 3.2 greatly improves [17, Theorem 4.1] and [18, Theorem 3.1].

\section{Acknowledgments}

The authors thank the referees for valuable comments and suggestions which improved their paper.

\section{References}

[1] R.P. Agarwal, D. O'Regan, M.-A. Taoudi, Fixed point theorems for ws-compact mappings in Banach spaces, Fixed Point Theory Appl. 2010, Article ID 183596 (2010) 13 pages.

[2] R.P. Agarwal, D. O'Regan, M.-A. Taoudi, Fixed point theorems for convex-power condensing operators relative to the weak topology and applications to Volterra integral equations, J. Int. Eq. Appl. 24 (2) (2012) 167-181. 
[3] O. Arino, S. Gautier, J.P. Penot, A fixed point theorem for sequentially continuous mappings with applications to ordinary differential equations, Funkc. Ekvac. 27 (1984) 273-279.

[4] J. Appell, The superposition operator in function spaces - a survey, Expo. Math. 6 (1988) 209-270.

[5] J. Banaś, J. Rivero, On measures of weak noncompactness, Ann. Mat. Pura Appl. 151 (1988) 213-224.

[6] J. Banaś, Z. Knap, Measure of weak noncompactness and nonlinear integral equations of convolution type, J. Math. Anal. Appl. 146 (1990) 353-362.

[7] J. Banaś, Z. Knap, Integrable solutions of a functional-integral equation, Rev. Mat. Univ. Complut. Madrid 2 (1) (1989) 31-38.

[8] J. Banaś, Integrable solutions of Hammerstein and Urysohn integral equations, J. Austral. Math. Soc. Ser. A 46 (1) (1989) 61-68.

[9] J. Banaś, M.-A. Taoudi, Fixed points and solutions of operator equations for the weak topology in Banach algebras, Taiwanese Journal of Mathematics 18 (2014) 871-893.

[10] C.S. Barroso, Krasnosel'skii's fixed point theorem for weakly continuous maps, Nonlinear Analysis 55 (1) (2003) 25-31.

[11] C.S. Barroso, E.V. Teixeira, A topological and geometric approach to fixed points results for sum of operators and applications, Nonlin. Anal. 60 (4) (2005) 625650 .

[12] A. Bellour, D. O'Regan, M.-A. Taoudi, On the existence of integrable solutions for a nonlinear quadratic integral equation, J. Appl. Math. Comput. 46 (1-2) (2014) 67-77.

[13] A. Bellour, M. Bousselsal, M.-A. Taoudi, Integrable solutions of a nonlinear integral equation related to some epidemic models, Glasnik Matematicki 49 (69) (2014) 395-406.

[14] A. Chlebowicz, M-A. Taoudi, Measures of weak noncompactness and fixed points, in: Advances in Nonlinear Analysis via the Concept of Measure of Noncompactness, Springer, Singapore, 2017, 247-296.

[15] F.S. De Blasi, On a property of the unit sphere in Banach spaces, Bull. Math. Soc. Sci. Math. Roum. 21 (1977) 259-262.

[16] S. Djebali, Z. Sahnoun, Nonlinear alternatives of Schauder and Krasnosel'skij types with applications to Hammerstein integral equations in $L^{1}$ spaces, J. Differential Equations 249 (9) (2010) 2061-2075. 
[17] Y. Du, Fixed points of increasing operators in ordered Banach spaces and applications, Applicable Analysis 38 (1990) 1-20.

[18] S.W. Du, V. Lakshmikantham, Monotone iterative technique for differential equations in a Banach space, J. Math. Anal. Appl. 87 (2) (1982) 454-459.

[19] N. Dunford, J.T. Schwartz, Linear Operators, Part I: General Theory, Interscience Publishers, New York, 1958.

[20] J. Garcia-Falset, Existence of fixed points and measure of weak noncompactness, Nonlin. Anal. 71 (2009) 2625-2633.

[21] J. Garcia-Falset, Existence of fixed points for the sum of two operators, Math. Nachr. 283 (12) (2010) 1736-1757.

[22] J. Garcia-Falset, K. Latrach, E. Moreno-Galvez, M.-A. Taoudi, SchaeferKrasnoselskii fixed point theorems using a usual measure of weak noncompactness, J. Differential Equations 252 (5) (2012) 3436-3452.

[23] D. Guo, Y.J. Chow, J. Zhu, Partial Ordering Methods in Nonlinear Problems, Nova Publishers, 2004.

[24] D.J. Guo, J.X. Sun, Z.L. Liu, The functional methods in nonlinear differential equation, Shandong Technical and Science Press (in chinese) (2006) 1-6.

[25] S. Heikkila, V. Lakshmikantham, Monotone Iterative Techniques for Discontinuous Nonlinear Differential Equations, CRC Press, 1994.

[26] N. Hussain, M.-A. Taoudi, Fixed point theorems for multivalued mappings in ordered Banach spaces with application to integral inclusions, Fixed Point Theory Appl. (2016) 2016:65.

[27] Y. Li, Z. Liu, Monotone iterative technique for addressing impulsive integrodifferential equations in Banach spaces, Nonlinear Anal. 66 (1) (2007) 83-92.

[28] E. Liz, Monotone iterative techniques in ordered Banach spaces, Proceedings of the Second World Congress of Nonlinear Analysts, Part 8 (Athens, 1996), Nonlinear Anal. 30 (8) (1997) 5179-5190.

[29] K. Latrach, M.-A. Taoudi, A. Zeghal, Some fixed point theorems of the Schauder and Krasnosel'skii type and application to nonlinear transport equations, J. Differential Equations 221 (1) (2006) 256-271.

[30] K. Latrach, M.-A. Taoudi, Existence results for a generalized nonlinear Hammerstein equation on $L^{1}$-spaces, Nonlin. Anal. 66 (2007) 2325-2333.

[31] A.R. Mitchell, C.K.L. Smith, An existence theorem for weak solutions of differential equations in Banach spaces, in: Nonlinear Equations in Abstract Spaces, (edited by V. Lakshmikantham), Academic Press, 1978, 387-404. 
[32] J. Sun, X, Zhang, The fixed point theorem of convex-power condensing operator and applications to abstract semilinear evolution equations, Acta Math. Sinica (in chinese) 48 (2005) 339-446.

[33] M.-A. Taoudi, Integrable solutions of a nonlinear functional integral equation on an unbounded interval, Nonlin. Anal. 71 (2009) 4131-4136.

[34] M.-A. Taoudi, Krasnosel'skii type fixed point theorems under weak topology features, Nonlinear Anal. 72 (1) (2010) 478-482.

[35] K. Yosida, Functional Analysis, Springer-Verlag, Berlin, 1965.

\section{DOI: $10.7862 /$ rf.2019.1}

\section{Abdullah Alahmari}

email: aaahmari@uqu.edu.sa

ORCID: 0000-0002-9596-910X

Department of Mathematics

College of Applied Sciences

P. O. Box 715, Makkah 21955

KSA

\section{Mohamed Mabrouk}

email: Mohamed.Mabrouk@fsg.rnu.tn

Department of Mathematics

College of Applied Sciences

P. O. Box 715, Makkah 21955

KSA

Department of Mathematics

Faculty of Sciences of Gabès

University of Gabès

Cité Erriadh, 6072 Zrig, Gabès

TUNISIA

\section{Mohamed-Aziz Taoudi}

email: a.taoudi@uca.ma

ORCID: 0000-0002-8851-8714

National School of Applied Sciences

Cadi Ayyad University

Marrakech

MOROCCO 